\title{
A Multi Agent System for Hospital Organization
}

\author{
Hanen Jemal, Zied Kechaou, Mounir Ben Ayed, and Adel M. Alimi
}

\begin{abstract}
In some countries the domain of medical care is characterized by difficulty, dynamism and diversity. In the $21^{\text {st }}$ century healthcare represents different challenges (increasing cost of care, population growth and lack of caregivers). For that, cooperation in the Agent Technology can provide better healthcare than the traditional medical system. In fact, Intelligent Agents properties (sociability, proactivity, autonomy) and the features of Multi Agent Systems (management of distributed information, communication and cooperation between different entities) are a good option to solve several problems in the hospital organization. As examples of problems, which appear in the hospital, we cite: collaboration between hospital wards, elaborations of medical diagnostics, coordination among medical entities and the collection of information about patients etc. In this regard, we propose a new medical system called MMAS (Medical MultiAgent System). The proposed system is complex; it integrates Multi-Agent System in healthcare in order to make care as efficient as possible.
\end{abstract}

Index Terms-Healthcare, agent technology, multi agent system, medical-multi-agent system.

\section{INTRODUCTION}

Since 2012 health service in Tunisia has grown into a more fundamental field in the Tunisian economy. The budget of the Tunisian healthcare sector reached $15 \%$ of the global budget. Thus, expenses in healthcare are an important part of macroeconomics costs. But the healthcare sector still has many problems (qualitative and qualitative): limited number of hospitals in the inner cities, poor infrastructure, lack of means and equipment, management of staff (recruitment, promotion, etc.), inadequate budget allocations, weak management of departments and low productivity of the current system.

The hospitals are confronted with difficulties which sometimes hinder their operations. Currently, the information systems in hospitals are not able to deal with the different problems such as coordination, cooperation and communication between all actors and different services which are not adequately conducted "Fig. 1". Also, identification and localization of equipment are still not handy in an automated way.

Several factors contribute to this unsatisfactory situation. Among the most important ones, it was noted:

1) Inadequate regulatory framework for hospitals: mission, functions, typology, etc are not clearly defined, despite the existence of some related legal texts.

2) Inadequate equipment and poor maintenance.

Manuscript received July 14, 2014; revised September 28, 2014.

The authors are with National School of Engineers (ENIS), REGIM: REsearch Group on Intelligent Machines, BP 1173, Sfax, 3038, Tunisia (email: \{hanen.jemal, zied.kechaou, mounir.benayed, adel.alimi\} @ieee.org).
These two factors explain why some hospital activities (surgery, for example) do not exist in some regional hospitals.

Thus, it is expected that information technology will play a role in reducing expenses and stabilizing healthcare budgets. The spread of computer sciences can help the challenges in using IT (Information Technology) for healthcare. Researchers will need to combine computer science and biomedical engineering to meet specific challenges in healthcare.

In fact, thanks to their major characteristics such as reactivity, autonomy, sociability and pro-activity, Multi Agent System (MAS) seems to be adapted to consider these loopholes.

In this article, a new Medical Multi-agent system called MMAS (Medical Multi-agent System) is proposed. In the hospital, several types of medical problems can be solved by agents. As examples of problems, which emerge in the hospital, we mention: collaboration between hospital wards, elaborations of diagnostics, the collection of information about patients etc. The adaptation of cooperative MAS can solve these problems.

The motivations underlying the use of MAS in the hospital consist in the several aspects that the agents can make during the realization of tasks are:

- MAS present the opportunity to decompose the problem: Agents working as a team specialized for defining and integrating information of patient in medical diagnostics.

- Coordination between different units and between actors participating in treatment process and optimized exchange of data between these units is one of the main challenges of MAS

- The agents negotiate autonomously in order to find equipments and information in order to reach an efficient resource.

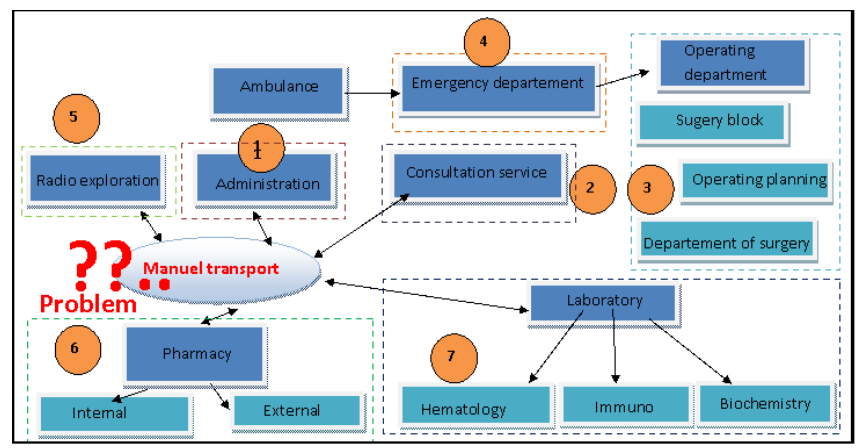

Fig. 1. The problem of coordination between seven services.

MAS' technology is a solution to meet requirements coming from complex and dynamic environment of hospital. Due to its flexibility and adaptability, an agent in health care, and in particular in the hospital logistics, was chosen.

Using MAS technology can also provide personalized assistance for multiple distributed actors, e.g., patients, 
doctors, nurses and other medical staff. In fact, our solution improves relationships between patient and caregivers.

From our studies we have derived various objectives for our research such as:

- Give patients, medical staff, hospitals and life-sciences researcher's better ways to interact in real-time, to communicate and manage information.

- Improve automation of healthcare domain.

- Increase access to information in hospital.

- Reduce costs by remotely accessing, raising productivity, minimizing the transfer of patients (Patients were able to choose a hospital, the date and time of their appointment also involve patient in discussions and decisions about their health care).

- Management of resources (human, supplies, operating room);

Finally, our vision is a to produce a connected healthcare system "Fig. 2" in order to improve the access to care, the productivity of medical staff and the quality of healthcare.

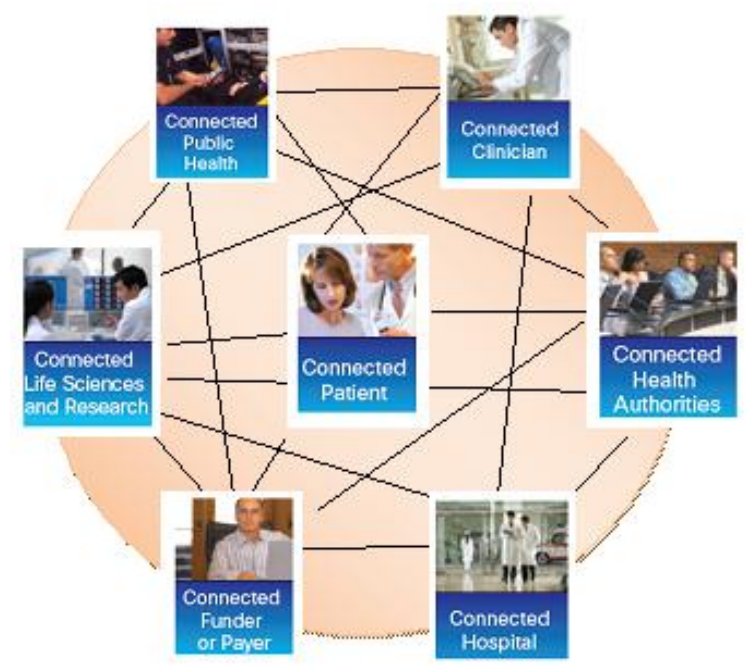

Fig. 2. Our connected healthcare ecosystem.

Our paper includes six parts. At first we started with an introduction, subsequently Section II represents a scientific state of art of the Multi Agent System in healthcare. Then we present our proposed architecture.

In Section IV we describe the implementation and results. Section V concerns the discussion and finally we present a conclusion.

\section{Multi AgENT System IN HeAlthCARE}

This study begins with presenting the relevant literature with view to proposing an integrated architecture to study the important factors affecting the decision to introduce MAS technology in the hospital industry.

The agents represent a very talented research in medical diagnoses and medical tasks. Several applications of the agents for healthcare were grouped into five fields: i) Medical data management, ii) Decision support systems, iii) Planning and resource allocation, iiii) Remote care and v) composite systems.

\section{A. Medical Data Management}

It presents all systems focused on the management, retrieval and processing of health data (e.g. EHR Electronic
Health Records) such as:

- The National electronic Library for Health $(\mathrm{NeLH})$ is a governmental project from National Health Service (NHS) from UK. NeLH introduce a portal to recover medical information via Internet [1]. The NeLH system contains network agents in order to update information, management and automation of the documents.

- The Virtual Electronic Patient Record (VEPR) presents a collection of agents that integrate documents from several information departmental systems [2]. Pro-activity of agents is exploited to automatically discover, consult and retrieve patients' data

- Context aware Hospital Information System (CHIS) is a MAS system that provides intelligence and pro-activity to healthcare environments: agents observe and find users in the system [3].

\section{B. Decision Support Systems}

This presents all systems and approaches aimed to help and assist caregivers in the performance of medical tasks such as:

- Singh et al. [4] present an intelligent assistant agent in healthcare. This assistant was considered to recover and use knowledge in order to solve medical episodes. They use case reasoning in order to capture the experiential knowledge of healthcare [5].

- The HealthAgents system is the result of a funded research project [6]. The aim of this project is to create a network that contributes to the diagnosis of brain tumors.

- Health Care Services (HeCaSe2) is a distributed system to deliver healthcare services [7].

\section{Planning and Resource Allocation}

It presents all systems focused on the scheduling and planning of physical and human resources like:

- The working groups "hospital logistics" including the ADAPT-Project developed a system called AgentHospital [8] is tested for agent-based information systems in healthcare, supporting both the development and the evaluation of modeling and implementation. At the model level a framework for different partial models of healthcare is provided. At the implementation level, infrastructure services and multi agent-based modular healthcare services exist.

- CARREL is an Agent Mediated Electronic Institution for the distribution of organs and tissues for transplantation purposes [9]. CARREL assists caregivers in the decision making during the sharing and allocation of pieces for transplants.

- The Medical Information Agents (MIA) presents a MAS to solve planning problems in health care [10].

The main goal of this project is to design MAS to achieve efficient planning in the healthcare dynamic environment.

\section{Remote Care}

It presents approaches focused on the remote monitoring of patients such as:

- The Aingeru system supports intelligent, permanent and persistent monitoring of elderly people through the PDA data [11].

- Koutkias et al. [12] present a system to ensure monitoring, surveillance and educational services for the management of chronic diseases. The proposed MAS aim to improve the performance by incorporating rule-based knowledge. 


\section{E. Composite Systems}

It presents all systems which offer complete solutions for healthcare.

- SHAREIT (Supported Human Autonomy for Recovery and Enhancement of Cognitive and motor disabilities using Agent Technologies) is a MAS which integrates technologies to build tools for users with sensor [13].

- Knowledge-Based Home Care e-Services for an Ageing Europe (K4Care) is a Home Care multi agent system model that provides e-services to caregivers. K4Care uses MAS technology to perform management of data [14].

- Geriatric Ambient Intelligence (GerAmI) is an intelligent supervision system delivering physical and cognitive support [15].The major goal is to maintain elderly and Alzheimer patients in all aspects of daily life.

- Haeng-Kon Kim in 2013 presents a multi-agent system based proactive u-healthcare system which incorporates different functions designed to resolve problems for the sake of rapid and efficient mobile u-healthcare agents. The proposed system allows the system itself to recognize and identify u-healthcare domain problems arising [16].

- In 2013 Mutingi and Mbohwa propose homecare multiagent system architecture in order to make decision with multiple objectives. The system integrates MAS based on Genetic Algorithm and Web Services that provide decisions in a dynamic multiple-objective environment. The proposed architecture consists of a number of agents that coordinated through efficient communication in homecare dynamic environments [17]. Table I summarizes the existing works in MAS.

TABLE I: EXISTING MAS WORKS IN HEALTHCARE

\begin{tabular}{|c|c|}
\hline Fields & Systems \\
\hline \multirow{3}{*}{ Decision support systems } & Singh et al. 2005 \\
\hline & Hecase2 2008 \\
\hline & Health agents 2008 \\
\hline \multirow{2}{*}{ Remote care } & Aingeru 2007 \\
\hline & Koutkias et al. 2007 \\
\hline \multirow{3}{*}{$\begin{array}{c}\text { Planning and resources } \\
\text { Allocation }\end{array}$} & MIA 2005 \\
\hline & CARREL 2008 \\
\hline & Agent Hospital 2008 \\
\hline \multirow{3}{*}{ Medical data management } & NeLH 2005 \\
\hline & VEPR 2006 \\
\hline & CHIS 2008 \\
\hline \multirow{5}{*}{ Composite systems } & SHAREIT 2008 \\
\hline & Ger Ami 2008 \\
\hline & K4Care 2009 \\
\hline & Mutingi and Mbohwa 2013 \\
\hline & Haeng-Kon Kim 2013 \\
\hline
\end{tabular}

Several applications have been proposed throughout the healthcare domain and intelligent systems satisfaction; but still, each architecture provided partial components for healthcare. As a consequence, many points appear:

First, the diverse architectures are not efficient when the difficulty and size of the environment increases.

Second, there are many challenges in measuring the quality of care. Little architecture proposals have been made for an evaluation. Consequently, a qualitative approach verifying the optimal evaluation process would be conducted for a better approach to healthcare.

Therefore, this solution makes primordial progress in healthcare domain, but mobile and cloud computing is absent in the process of healthcare.

Our research aims to elaborate a MAS generic framework in distributed healthcare systems. In order to achieve this purpose, several tools like Web Service, Fuzzy Ontology, Social Network and Mobile Computing are used. In this paper, we will only detail the MAS. The agent approach has shown a potential for being deployed in different applications in healthcare services. Agents can maintain the autonomy of the collaborating participants, integrate disparate operating environments, and coordinate distributed data.

\section{Proposed ARChITECTURE}

Our hospital architecture hierarchically organizes component structure of agents; hence the agents of our hospital can be classified in two groups or layers:

- Intelligent agents or super agent (doctor, patient, nurses....)

- Swarm layer inspired from the Swarm Intelligence fields (applies the collective behavior of groups to resolve a problem.) such as office, medical materials ...

In this paper we present the interaction in an agent-based architecture for healthcare. This architecture is composed of a set of autonomous agents adapted to the interaction. This architecture includes different intelligent agents, representing a medical organization with different roles and communication patterns, and facilitates the interaction, the interoperability, and the accessibility to information. Agents are involved in functions. In particular, the knowledge is completely distributed between agents and thus assuring flexibility.

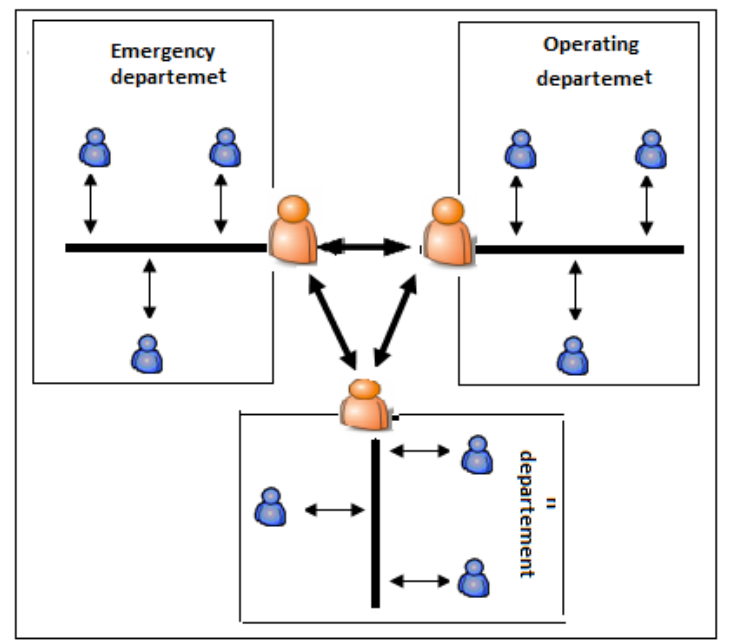

Fig. 3. Global Structure of Hospital.

: Agent's inter-department (Classified according to the two previous layers)

8: Agent's intra-department (Classified according to the two previous layers)

$\leftrightarrow \quad$ : Channels of communication between the agents

Dept i: Department i (Castro, radiology ....)

Each hospital is a set of departments "Fig. 3" based on the following conjunction: resources /interaction. We consider 
two types of interactions between agents: informational and physical. Resources can be of three types: human (patients, nurses, agents....), material (carriage, chair, dressing ...) and informational (patient records, schedule, cost ...).

The agents of our system and their roles are described below. Tasks assigned to each agent are specific to each model:

\section{Agent Doctor}

1): Ad (i) transmitted diagnoses to expert agents (Expert Decision System based on Intuitionist Fuzzy Logic)

2): Each expert agent Ax (i) consults diagnoses and conducts the analysis of the data

3): Each Ax (i) returns the answer of its examination

4): Ad (i) chooses the most adequate result.

- Gives the appropriate treatment to the patient

- Decides suitable treatment

- Gives a patient's treatment

Search agent

- Search available human (doctor) and physical resources

Patient agent

- Maintains and improve her health status.

- Makes self-management of his illness and detect the deterioration in his health status

- Chooses a healthcare center Nurse agent

- Locates wheelchair via RFID (Radio Frequency Identification), and automatically dispatches a porter to bring wheelchair to patient's location.

- Gives suitable treatment according to doctor directive

Access agent

- Locates wheelchair via RFID (Radio Frequency Identification), and automatically dispatches a porter to bring wheelchair to patient's location.

Discharge agent

- Responsible for patients discharge

- Refer the patient to another healthcare service or center

- Gives suitable treatment according to doctor directive

Service agent

- Responsible for delivering the services offered by the center.

- Manage and allocate human and physical resources between services

- Manages collaboration with other centers

Medical center administration agent

- Remote consultations with specialists via TV

- Shares EMR and images

Calendar agent

- Provides the list of the doctors available by consulting the calendar to associate the appointment

Emergency medical agent

- Uses communication radios to inform local hospital of incoming patient.

Security and safety agent

- Supervises patients in high menace areas along with sensing levels of biologicals and gases

- Monitors security of department and equipment

Agent test results

- Notifies the test results from the Hospital Lab and Radiology Department

Our aim is that agents work in the background to provide ambient intelligence to the users, who reside in the cloud environment i.e., the agents communicate with each other, acquire their behavior and receive information through medical cloud data.

\section{IMPLEMENTATION AND RESULTS}

\section{A. Implementation}

To implement our proposed application, we have chosen JADE (Java Agent Development Framework) as a software framework for developing MAS. JADE presents several advantages [18] such as Interoperability, Portability, Simplicity, Distributed, Programmable interface and FIPA (Foundation for Intelligent Physical Agents) protocols Library Standard. Also there are different reasons for using Jade in our application. This software supports the ontology tools and we can integrate it with Web technologies such as Web Service, Servlets and JSP.

We use JSP, Servlets, Jade and a Fuzzy logic system to introduce a fuzzy learning algorithm for expert agents ("Fig. 4" represents result of fuzzy analysis).

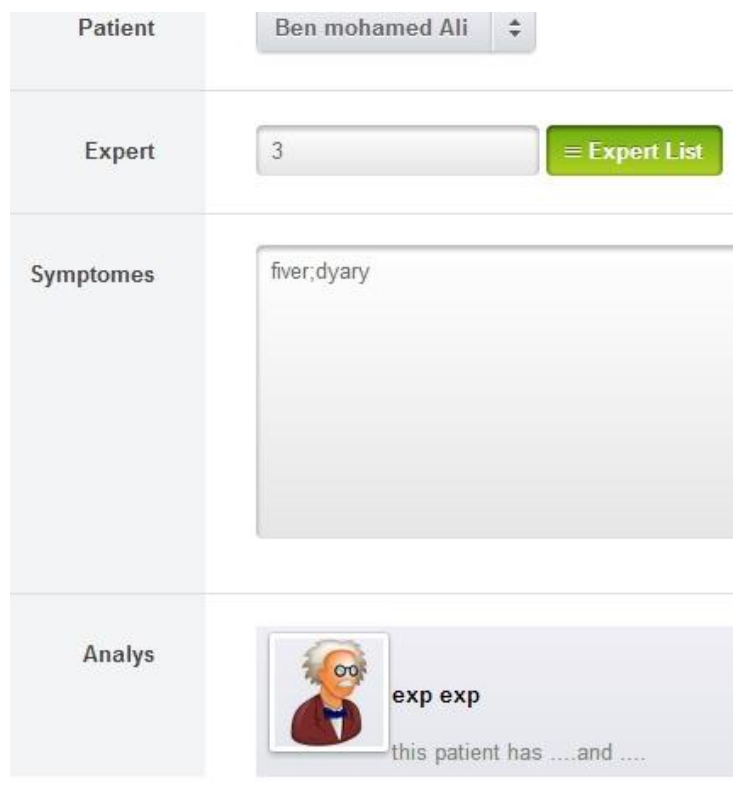

Fig. 4. Expert analysis.

The platform is a tool for several users:

1) Hospital staff: represent all the agents that are concerned in providing care to patients in the healthcare system. This community should be able to know and confirm the abstract representation of the theoretical model of the hospital as well as manipulate and utilize the results of the system. The main components of this category are:

- Medical personnel: physician, surgeon, radiologist, anesthetist, etc.

- Nursery personnel;

- Technicians: such as laboratory personnel

- Admission and discharge personnel;

- Medical support personnel: security, archive, supplying, cleaning, etc.

2) Team study like researchers, engineers.

3) Patients represent the main users of the healthcare system and the core of the care system.

4) Administrator of the application

\section{B. Results}

We achieve the implantation of several services such as: 
- Patient appointment: patient can choose a date for remote consultation "Fig. 5".

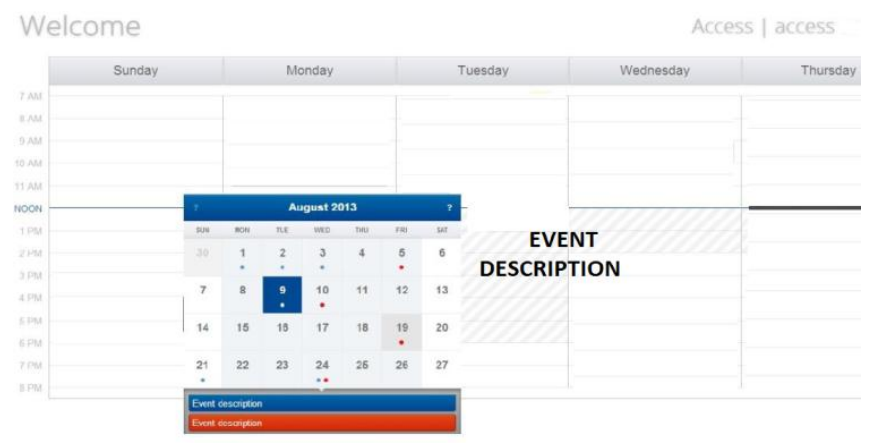

Fig. 5. Patient appointment.

- Remote consultation: patient can make consultation in real times by sending and receiving message from doctors.

- Resource allocation: doctors and patients can allocate resources at a distance (IRM, scanner...).

- Connection to cloud computing storage (e.g. patient health records): The system application allows caregivers to save and upload distributed medical data.

- Image viewing by supporting The DICOM medical image.

- Patient registration: patients can make registration remotely as well as choose the medical center and the suitable service (Pediatric, radiology...). "Fig. 6" presents a home interface, after authentication we can access according to the role (doctor, patient, nurse, expert...)

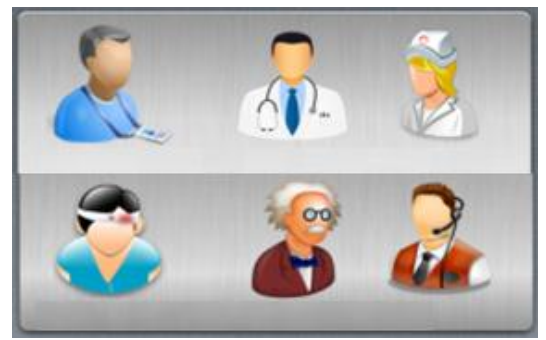

Fig. 6. The home interface.

\section{DISSCUSSION}

The proposed system is complex; the members cooperate in order to handle the difficulty of hospital problems. During the development of the prototype system, we came across several attractive points for multi agents. Here are the points which we came across while implementing the system:

- Extensibility: integration of new entities to cooperate with the existing ones.

- Portability: These are based on the use of the standard of communications such as ACL

- Performance: it is related to the intrinsic opportunities for agents (asynchrony and parallel execution).

- Robustness: these can be guaranteed by the adaptive capabilities of agents in the different situations.

\section{CONCLUSION}

The presented work is an integration of information technology in the health delivery process. Thus Intelligent Agents have a set of properties (sociability, proactivity, autonomy) that make them suitable to be used to solve many problems that appear in the healthcare domain. This basic property of Intelligent Agents and the features of Multi Agent Systems (management of distributed information, communication and cooperation between separate autonomous entities) suggest that they are a good option to solve the problems of coordination in the hospital organization.

As a future work, we plan to introduce several improvements or possible extensions to the architecture like the definition of a coherent ontology in the communication between agents in order to surmount the problem of the semantic interoperability. We also plan to implement a medical ontology and make a cloud simulation.

\section{ACKNOWLEDGEMENTS}

The author would like to acknowledge the financial support of this work by grants from the General Direction of Scientific Research and Technological Renovation (DGRSRT), Tunisia, under the ARUB program LR11ES48.

\section{REFERENCES}

[1] P. Kostkova, J. ManiSaada, G. Madle, and J. R. Weinberg, Applications of software Agent Technology in the Health Care Domain, Birkhäuser Verlag, Basel, Switzerland, 2003, Ch. AgentBased Uptodate Data Management in National Electronic Library for Communicable Disease, pp. 105-124.

[2] P. V. Marques, S. Robles, J. Cucurull, R. C. Correia, G. Navarro, and R. Marti, "Secure integration of distributed medical data using mobile agents," IEEE Intelligent Systems, vol. 21. no. 6, pp. 47-54, 2006.

[3] M. Tentori, J. Favela, and M. Rodriguez, "Privacyaware autonomous agents for pervasive healthcare," IEEE Intelligent Systems, vol. 21, no. 6 , pp. 55-62, 2006.

[4] S. Singh, B. Ikhwan, F. Haron, and C. H. Yong, "Architecture of agentbased healthcare intelligent assistant on grid environment," in Proc. the 5th International Conference on Parallel and Distributed Computing: Applications and Technologies, K. M. Liew, H. Shen, S. See, W. Cai, P. Fan, and S. Horiguchi, Eds. vol. 3320 of LNCS, Singapore: Springer, 2005, pp. 58-61.

[5] H. GonzálezVélez, M. Mier, M. JuliàSapé, T. N. Arvanitis, J. M. GarcíaGómez, M. Robles, P. H. Lewis, S. Dasmahapatra, D. Dupplaw, A. Peet, C. Arús, B. Celda, S. V. Huffe, and M. LluchAriet, "HealthAgents: Distributed multiagent brain tumor diagnosis and prognosis," Applied Intelligence, vol. 30, no. 3, pp. 191-202, 2009.

[6] D. Isern and A. Moreno, "Computerbased execution of clinical guidelines: A review," International Journal of Medical Informatics, vol. 77 , no. 12 , pp. $787-808,2008$.

[7] S. Kirn, C. Anhalt, H. Krcmar, and A. Schweiger, "Multiagent engineering: Theory and applications in enterprises," International Handbook on Information Systems, Springer, Berlin-Heidelberg, Ch. Agent. Hospital-Health Care Applications of Intelligent Agents, pp. 199-220, 2006.

[8] U. Cortés, J. VázquezSalceda, A. LópezNavidad, and F. Caballero, "UCTx: A multiagent approach to model a transplant coordination unit," Journal of Applied Intelligence, vol. 20, no. 1, pp. 59-70, 2004.

[9] L. Braun, F. Wiesman, J. van der Herik, and A. Hasman, "Agent support in medical information retrieval," in Proc. the 3rd Workshop on Agents Applied in Health Care, Eds. U. Cortés, J. Fox, A. Moreno, J. Nealon, AAAI, Edimburgh, Scotland, 2005.

[10] M. I. Bagüés, J. Bermúdez, A. Illarramendi, A. Tablado, and A. Gon i, "An innovative system that runs on a PDA for a continuous monitoring of people," in Proc. the 19th IEEE Symposium on Computer Based Medical Systems, P. Kokol, T. WelzerDru `zovec, D. MiceticTurk, M. Zorman, Eds. Maribor, Slovenia: IEEE Press, 2006, pp. 151-156.

[11] V. Koutkias, I. Chouvarda, and N. Maglaveras, "A multiagent system enhancing homecare health services for chronic disease management," IEEE Transactions on Information Technology in Biomedicine, vol. 9, no. 4, pp. 528-537, 2005. 
[12] L. Cervantes, Y. S. Lee, H. Yang, S. H. Ko, and J. Lee, "Agent based intelligent decision support for the home healthcare environment," in Proc. the 1st International Conference on Advances in Hybrid InformationTechnology, M. S. Szczuka, D. Howard, D. Slezak, H. K. Kim, T. H. Kim, I. S. Ko, G. Lee, P. M. Sloot, Eds. vol. 4413 of LNAI, Jeju Island, Korea: Springer, 2007, vol. 4413, pp. 414-424.

[13] U. Cortés, R. Annicchiarico, C. Urdiales, C. Barrué, A. Martínez, A Villar, and C. Caltagirone, "Agent Technology and eHealth," Whitestein Series in Software Agent Technologies and Autonomic Computing, Birkhäuser Verlag, Basel, Switzerland, Ch. Supported Human Autonomy for Recovery and Enhancement of Cognitive and Motor Abilities Using Agent Technologies, pp. 117-140, 2008.

[14] D. Isern, A. Moreno, D. Sánchez, A. Hajnal, G. Pedone, and L. Z. Varga, "Agentbased execution of personalised home care treatments," Journal of Applied Intelligence, vol. 34, issue 2, pp. 155-180, April 2011.

[15] J. M. Corchado, J. Bajo, and A. Abraham, "GerAmI: Improving healthcare delivery in geriatric residences," IEEE Intelligent Systems, vol. 23, pp. 19-25, 2008

[16] H. K. Kim, "Convergence agent model for developing u-healthcare systems," School of Information Technology, Catholic University of Deagu 712702, Rep. of Korea, 2013.

[17] M. Mutingi and C. Mbohwa, "A home healthcare multi-agent system in a multi-objective environment," SAIIE25 Proceedings, Stellenbosch, South Africa SAIIE 636-1, 9-11, July 2013

[18] JADE Home Page. (2004). Java Agent DEvelopment Framework (JADE). [Online]. Available: http://jade.tilab.com/

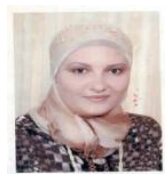

H. Jemal is a member of IEEE since 2011, who graduated in computer science engineering in 2009.

She is a PhD student in National School of Engineers (ENIS), REGIM: REsearch Group on Intelligent Machine BP 1173, Sfax, 3038, Tunisia.

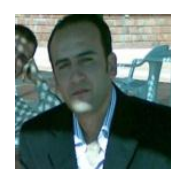

Z. Kechaou is a member of IEEE, who is an assistant in Higher Institute of Transport and Logistics, University of Sousse.

$\mathrm{He}$ is a member of REsearch Group on Intelligent Machine BP 1173, Sfax, 3038, Tunisia.

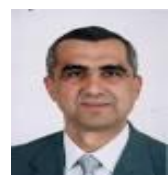

M. Ben Ayed is an assistant professor in the computer Science Department of the Faculty of Science, University of Sfax. He teaches DBMS, data warehouse and data mining. His research activities concern DSS based on a KDD process. Most of his research works are applied in health care domain.

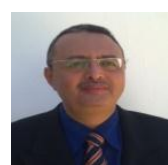

Adel M. Alimi graduated in electrical engineering in 1990. He obtained a PhD and then an HDR both in electrical \& computer engineering in 1995 and 2000 respectively. $\mathrm{He}$ is a full professor in electrical engineering at the University of Sfax since 2006. Prof. Alimi is the founder and director of the REGIM-Lab. on intelligent machines. 